TRANSACTIONS OF THE

AMERICAN MATHEMATICAL SOCIETY

Volume 364, Number 5, May 2012, Pages 2521-2538

S 0002-9947(2011)05384-5

Article electronically published on December 16, 2011

\title{
THE FINITE INTERVALS OF THE MUCHNIK LATTICE
}

\author{
SEBASTIAAN A. TERWIJN
}

\begin{abstract}
We characterize the finite intervals of the Muchnik lattice by proving that they form a certain proper subclass of the finite distributive lattices. We also discuss infinite intervals, mainly to conclude that much more is possible here than for the related Medvedev lattice.
\end{abstract}

\section{INTRODUCTION}

The Medvedev lattice and the Muchnik lattice are structures from computability theory that were originally defined for their connections with constructive logic, but that are of independent interest as well. Both can be seen as generalizations of the Turing degrees, and for example when Muchnik presented his solution to Post's problem he phrased it as a result about the Medvedev lattice. In Terwijn [21] the structure of the Medvedev lattice $\mathfrak{M}$ was investigated, and it was proven there that the finite intervals of $\mathfrak{M}$ are precisely the finite Boolean algebras, and that the infinite intervals of $\mathfrak{M}$ all have cardinality $2^{2^{\aleph_{0}}}$ (cf. Theorem 1.5 below). It was noted there that this strong dichotomy does not hold for the Muchnik lattice $\mathfrak{M}_{w}$, and that there are many more possibilities for intervals in $\mathfrak{M}_{w}$, both for the finite and for the infinite ones. In this paper we characterize the finite intervals of $\mathfrak{M}_{w}$ by proving that they are a certain subclass of the finite distributive lattices that can be described using elementary lattice theory. In the rest of this section we will repeat the necessary definitions and list some further preliminaries.

The Medvedev lattice, introduced by Medvedev [8, is a particular way of specifying Kolmogorov's idea of a calculus of problems. Let $\omega$ denote the natural numbers and let $\omega^{\omega}$ be the set of all functions from $\omega$ to $\omega$ (Baire space). A mass problem is a subset of $\omega^{\omega}$. Every mass problem is associated with the "problem" of producing an element of it. A mass problem $\mathcal{A}$ Medvedev reduces to a mass problem $\mathcal{B}$, denoted $\mathcal{A} \leqslant{ }_{M} \mathcal{B}$, if there is a partial computable functional $\Psi: \omega^{\omega} \rightarrow \omega^{\omega}$ defined on all of $\mathcal{B}$ such that $\Psi(\mathcal{B}) \subseteq \mathcal{A}$. (For background on computable functionals we refer the reader to 13 or [11.) That is, $\Psi$ is a uniformly effective method for transforming solutions to $\mathcal{B}$ into solutions to $\mathcal{A}$. The relation $\leqslant_{M}$ induces an equivalence relation on mass problems: $\mathcal{A} \equiv_{M} \mathcal{B}$ if $\mathcal{A} \leqslant_{M} \mathcal{B}$ and $\mathcal{B} \leqslant_{M} \mathcal{A}$. The equivalence class of $\mathcal{A}$ is denoted by $\operatorname{deg}_{M}(\mathcal{A})$ and is called the Medvedev degree of $\mathcal{A}$. We denote Medvedev degrees by boldface symbols. There is a smallest Medvedev degree, denoted by $\mathbf{0}$, namely the degree of any mass problem containing a computable function, and there is a largest degree $\mathbf{1}$, the degree of the empty mass problem, of which it is absolutely impossible to produce an element. A meet operator $\times$ and a

Received by the editors April 6, 2009 and, in revised form, May 24, 2010.

2010 Mathematics Subject Classification. Primary 03D30, 03G10, 06B05, 06D20.

Key words and phrases. Muchnik lattice, finite distributive lattices, Turing degrees.

This research was supported by the Austrian Science Fund FWF under project P18713-N18.

(C)2011 American Mathematical Society

Reverts to public domain 28 years from publication 
join operator + are defined on mass problems as follows: For functions $f$ and $g$, as usual define the function $f \oplus g$ by $f \oplus g(2 x)=f(x)$ and $f \oplus g(2 x+1)=g(x)$. Let $\widehat{n \mathcal{A}}=\{\widehat{n f f}: f \in \mathcal{A}\}$, where ${ }^{\widehat{y}}$ denotes concatenation (either of two finite strings or of a finite and an infinite string, as in this case). Define

$$
\mathcal{A}+\mathcal{B}=\{f \oplus g: f \in \mathcal{A} \wedge g \in B\}
$$

and

$$
\mathcal{A} \times \mathcal{B}=0^{\wedge} \mathcal{A} \cup 1^{\wedge} \mathcal{B}
$$

The definitions of the ordering $\leqslant_{M}$ and of the operations + and $\times$ on mass problems extend to the Medvedev degrees in the obvious way. The structure $\mathfrak{M}$ of all Medvedev degrees, ordered by $\leqslant_{M}$ and together with + and $\times$ is a distributive lattice (Medvedev [8]).

The Muchnik lattice, introduced by Muchnik [10], is a nonuniform variant of the Medvedev lattice. It is the structure $\mathfrak{M}_{w}$ resulting from the reduction relation on mass problems defined by

$$
\mathcal{A} \leqslant_{w} \mathcal{B} \equiv(\forall f \in \mathcal{B})(\exists g \in \mathcal{A})\left[g \leqslant_{T} f\right] .
$$

(The "w" stands for "weak".) That is, every solution to the mass problem $\mathcal{B}$ can compute a solution to the mass problem $\mathcal{A}$, but not necessarily in a uniform way. The lattice operations in $\mathfrak{M}_{w}$ are defined from the same operations + and $\times$ on mass problems as for $\mathfrak{M}$. Notice that $\mathcal{A} \times \mathcal{B}$ in $\mathfrak{M}_{w}$ simplifies to $\mathcal{A} \cup \mathcal{B}$. Also 0 and $\mathbf{1}$ in $\mathfrak{M}_{w}$ are defined in the same way. It is easy to check that $\mathfrak{M}_{w}$ is again a distributive lattice.

An M-degree is a Muchnik degree if it contains a mass problem that is upwards closed under Turing reducibility $\leqslant_{T}$. The Muchnik degrees of $\mathfrak{M}$ form a substructure that is isomorphic to $\mathfrak{M}_{w}$ as an upper semilattice (meets are not necessarily the same in $\mathfrak{M}$ as in $\mathfrak{M}_{w}$ ). For any mass problem $\mathcal{A}$, let $C(\mathcal{A})$ denote the upward closure of $\mathcal{A}$ under $\leqslant_{T}$. We have the following embeddings of upper semilattices:

$\begin{array}{ccccc}\mathcal{D}_{T} & \hookrightarrow & \mathfrak{M}_{w} & \hookrightarrow & \begin{array}{c}\mathfrak{M} \\ \text { Muchnik degrees }\end{array} \\ \text { Turing degrees } & & & \text { Medvedev degrees } \\ \operatorname{deg}_{T}(f) & \longmapsto & \operatorname{deg}_{w}(\{f\}) & & \\ & & \operatorname{deg}_{w}(\mathcal{A}) & \longmapsto & \operatorname{deg}_{M}(C(\mathcal{A})) .\end{array}$

That the embedding $\mathfrak{M}_{w} \hookrightarrow \mathfrak{M}$ above is an embedding of upper semilattices, but not preserving infima, was observed in Sorbi [15, Proposition 3.8]. That the embedding $\mathcal{D}_{T} \hookrightarrow \mathfrak{M}_{w}$ above is an embedding of upper semilattices (not preserving infima) follows from [15, Theorem 2.8].

More discussion about the elementary properties of $\mathfrak{M}$ and $\mathfrak{M}_{w}$ can be found in Rogers' textbook [13] and the survey paper by Sorbi [17]. Previous results about embeddings of lattices and algebras into $\mathfrak{M}$ and $\mathfrak{M}_{w}$ can be found in Sorbi [15, 16]. Binns and Simpson [2] contains results about lattice embeddings into the lattice of $\Pi_{1}^{0}$-classes under $\leqslant_{M}$ and $\leqslant_{w}$.

Our notation is mostly standard and follows Odifreddi [11. $\Phi_{e}$ is the $e$-th partial computable functional. For countable sets $I \subseteq \omega$ and mass problems $\mathcal{A}_{i}, i \in I$, we have the meet operator

$$
\prod_{i \in I} \mathcal{A}_{i}=\left\{i^{\wedge} f: i \in I \wedge f \in \mathcal{A}_{i}\right\} .
$$


Note that for finite $I$ this is M-equivalent to an iteration of the meet operator $\times$. If $a \leqslant b$ in a given partial order, we use the interval notation $[a, b]=\{x: a \leqslant x \leqslant b\}$. Similarly $(a, b)$ denotes an interval without endpoints, and $(a]$ denotes the set $\{x$ : $x \leqslant a\}$. We say that $b$ covers $a$ if $b>a$ and there is no $x$ with $a<x<b$. We should warn the reader that in order to save notation we often identify degrees (Turing, Medvedev, and Muchnik) with their representatives. E.g. if it is understood that we are working in $\mathfrak{M}_{w}$ we sometimes write $[\mathcal{A}, \mathcal{B}]$ for the interval $\left[\operatorname{deg}_{w}(\mathcal{A}), \operatorname{deg}_{w}(\mathcal{B})\right]$.

In the final section of 21] some consequences of the results of that paper for the Muchnik lattice $\mathfrak{M}_{w}$ were listed. Some of these consequences were:

- In contrast to $\mathfrak{M}$, the lattice $\mathfrak{M}_{w}$ contains nonempty linear intervals.

- Every finite Boolean algebra is isomorphic to an interval of $\mathfrak{M}_{w}$.

- Whereas in $\mathfrak{M}$ only countable Boolean algebras can be embedded, the dual of $\mathcal{P}\left(2^{\omega}\right)$ is embeddable into $\mathfrak{M}_{w}$ as a Boolean algebra.

A Medvedev or Muchnik degree is a degree of solvability if it contains a singleton mass problem. When no confusion arises we sometimes also call these degrees Turing degrees. For every degree of solvability $\mathbf{S}$ there is a unique minimal Mdegree $>\mathbf{S}$ that is denoted by $\mathbf{S}^{\prime}$ (cf. Medvedev [8]). If $\mathbf{S}=\operatorname{deg}_{M}(\{f\})$, then $\mathbf{S}^{\prime}$ is the degree of the mass problem

$$
\{f\}^{\prime}=\left\{\widehat{n} g: f<_{T} g \wedge \Phi_{n}(g)=f\right\} .
$$

Note that for any computable $f$ the set $\{f\}^{\prime}$ is M-equivalent to the set of all noncomputable functions. We will also denote this set by $0^{\prime}$. Note further that for any $f$ we have $\{f\}^{\prime} \equiv_{w}\left\{g \in \omega^{\omega}: f<_{T} g\right\}$ so that in $\mathfrak{M}_{w}$ we can use this simplified version of $\{f\}^{\prime}$. Dyment [4 proved that the degrees of solvability are precisely characterized by the existence of such an $\mathbf{S}^{\prime} 11$ Namely, the degrees of solvability are first-order definable (both in $\mathfrak{M}$ and in $\mathfrak{M}_{w}$ ) by the formula

$$
\phi(x)=\exists y(x<y \wedge \forall z(x<z \rightarrow y \leqslant z)) .
$$

Thus the Turing degrees form a first-order definable substructure of both $\mathfrak{M}$ and $\mathfrak{M}_{w} 2$ This has many immediate corollaries, for example that the first-order theories of the structures $\left(\mathfrak{M}, \leqslant_{M}\right)$ and $\left(\mathfrak{M}_{w}, \leqslant_{w}\right)$ are undecidable 3

Theorem 1.1 (Dyment [4; cf. [21, Theorem 2.5]). For Medvedev degrees $\mathbf{A}$ and $\mathbf{B}$ with $\mathbf{A}<_{M} \mathbf{B}$ it holds that $(\mathbf{A}, \mathbf{B})=\emptyset$ if and only if there is a degree of solvability $\mathbf{S}$ such that $\mathbf{A} \equiv_{M} \mathbf{B} \times \mathbf{S}, \mathbf{B} \Varangle_{M} \mathbf{S}$, and $\mathbf{B} \leqslant_{M} \mathbf{S}^{\prime}$.

Theorem 1.1 also holds for $\mathfrak{M}_{w}$, with a much easier proof. We will include a proof here, as a warm-up for Section 3 .

Lemma 1.2. Suppose that $\mathcal{A}$ and $\mathcal{B}$ satisfy

$$
\forall \mathcal{C} \subseteq \mathcal{A} \text { finite }\left(\mathcal{B} \times \mathcal{C} \nless{ }_{w} \mathcal{A}\right)
$$

\footnotetext{
${ }^{1}$ The characterization was for $\mathfrak{M}$, but the same proof works for $\mathfrak{M}_{w}$, and is in fact easier. (Cf. Theorem 1.3 below.)

${ }^{2}$ That the formula $\phi$ indeed defines the Turing degrees in $\mathfrak{M}$ can also be seen using Theorem 1.1 as follows. If $\mathbf{A}$ is a Turing degree, then $\phi$ is satisfied by $\mathbf{A}^{\prime}$. Conversely, suppose $\mathbf{A}$ is not Turing and suppose for a contradiction that it satisfies $\phi$, as witnessed by $\mathbf{B}$. Then $(\mathbf{A}, \mathbf{B})=\emptyset$, so by Theorem 1.1 there is a Turing degree $\mathbf{S} \geqslant_{M} \mathbf{A}$ that satisfies the conditions from the theorem. But A is not Turing; hence $\mathbf{A} \not_{M} \mathbf{S}$, and hence $\mathbf{S} \geqslant_{M} \mathbf{B}$. This contradicts the condition $\mathbf{B} \Varangle_{M} \mathbf{S}$ from the theorem.

${ }^{3}$ Recently, Lewis, Nies, and Sorbi proved that the degree of both theories is in fact the same as that of third-order arithmetic. These results were obtained independently by Shafer.
} 
Then there exists $\mathcal{C} \geqslant_{w} \mathcal{A}$ such that $\mathcal{C} \Varangle_{w} \mathcal{B}$ and $\mathcal{B} \times \mathcal{C} \Varangle_{w} \mathcal{A}$. If moreover $\mathcal{A} \leqslant_{w} \mathcal{B}$, then the interval $(\mathcal{A}, \mathcal{B})$ is infinite.

Proof. Since from (1.1) it follows that $\mathcal{B} \mathbb{K}_{w} \mathcal{A}$, there is $f \in \mathcal{A}$ such that $\{f\} \ngtr_{w} \mathcal{B}$. Again by (1.1) we have that $\mathcal{B} \times\{f\} \mathbb{K}_{w} \mathcal{A}$, so we can take $\mathcal{C}=\{f\}$.

If in addition $\mathcal{A} \leqslant{ }_{w} \mathcal{B}$, then we have $\mathcal{A}<_{w} \mathcal{B} \times\{f\}<_{w} \mathcal{B}$. Since $\mathcal{A}$ and $\mathcal{B} \times\{f\}$ also satisfy (1.1) we can by iteration of the first part of the lemma obtain an infinite downward chain in $(\mathcal{A}, \mathcal{B})$.

Theorem 1.3 (Dyment's Theorem for $\mathfrak{M}_{w}$ ). For Muchnik degrees A and $\mathbf{B}$ with $\mathbf{A}<_{w} \mathbf{B}$ it holds that $(\mathbf{A}, \mathbf{B})=\emptyset$ if and only if there is a degree of solvability $\mathbf{S}$ such that $\mathbf{A} \equiv_{w} \mathbf{B} \times \mathbf{S}, \mathbf{B} \Varangle_{w} \mathbf{S}$, and $\mathbf{B} \leqslant{ }_{w} \mathbf{S}^{\prime}$.

Proof. (If) Suppose that $\mathbf{S}=\operatorname{deg}_{w}(\{f\})$ is as in the theorem and suppose that $\mathcal{A} \in \mathbf{A}, \mathcal{B} \in \mathbf{B}$, and $\mathcal{B} \times\{f\} \leqslant_{w} \mathcal{C} \leqslant_{w} \mathcal{B}$. If $\mathcal{C}$ does not contain any element of Turing degree $\operatorname{deg}_{T}(f)$, then it follows that $\mathcal{C} \geqslant{ }_{w} \mathcal{B} \times\{f\}^{\prime}$, because the elements of $\mathcal{C}$ that get sent to the $\{f\}$-side are all strictly above $f$, hence included in $\{f\}^{\prime}$. So in this case $\mathcal{C} \geqslant{ }_{w} \mathcal{B}$ by $\{f\}^{\prime} \geqslant{ }_{w} \mathcal{B}$.

Otherwise $\mathcal{C}$ contains an element of Turing degree $\operatorname{deg}_{T}(f)$, and consequently $\mathcal{C} \leqslant{ }_{w}\{f\}$. Hence $\mathcal{C} \leqslant_{w} \mathcal{B} \times\{f\} \equiv_{w} \mathcal{A}$.

(Only if) Suppose that $(\mathcal{A}, \mathcal{B})=\emptyset$. Then by Lemma 1.2. $\mathcal{A}$ and $\mathcal{B}$ do not satisfy condition (1.1); hence there is a finite set $\mathcal{C} \subseteq \mathcal{A}$ such that $\mathcal{B} \times \mathcal{C} \leqslant w \mathcal{A}$. There is also an $f \in \mathcal{C}$ such that $\{f\} \Varangle_{w} \mathcal{B}$, for otherwise we would have $\mathcal{A} \geqslant_{w} \mathcal{B}$. Because the interval is empty and $\mathcal{A} \leqslant w \mathcal{B} \times\{f\}<_{w} \mathcal{B}$ we must have $\mathcal{A} \equiv_{w} \mathcal{B} \times\{f\}$ since there is no other possibility for $\mathcal{B} \times\{f\}$. We also have $\mathcal{B} \times\{f\}^{\prime} \mathbb{w}_{w} \mathcal{A}$ because both $\{f\} \nexists_{w} \mathcal{B}$ and $\{f\} \nexists_{w}\{f\}^{\prime}$. Hence $\mathcal{B} \times\{f\}^{\prime} \equiv_{w} \mathcal{B}$, again by emptiness of the interval, and in particular $\{f\}^{\prime} \geqslant w \mathcal{B}$. So we can take $\mathbf{S}$ to be $\operatorname{deg}_{w}(\{f\})$.

Let $f$ and $g$ be T-incomparable. Then it follows from Theorem 1.3 that the interval $\left[\{f, g\},\{f\}^{\prime} \times\{g\}^{\prime}\right]$ in $\mathfrak{M}_{w}$ contains exactly two intermediate elements; cf. Figure 1.

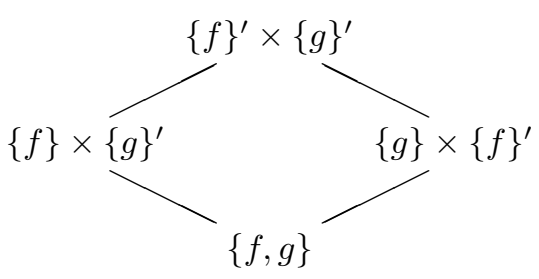

FiguRe 1

This can be generalized to obtain finite intervals of size $2^{n}$ for any $n$ as follows:

Theorem 1.4. Let $\mathcal{B}$ be any mass problem. Let $n \geqslant 1$ and let $f_{1}, \ldots, f_{n} \in \omega^{\omega}$ be $T$-incomparable such that $\left\{f_{i}\right\} \ngtr_{w} \mathcal{B}$ for every $i$. Then the interval

$$
\left[\mathcal{B} \times\left\{f_{1}, \ldots, f_{n}\right\}, \mathcal{B} \times\left\{f_{1}\right\}^{\prime} \times \ldots \times\left\{f_{n}\right\}^{\prime}\right]
$$

in $\mathfrak{M}_{w}$ is isomorphic to the finite Boolean algebra $2^{n}$ consisting of all subsets of $\{1, \ldots, n\}$.

Proof. This was proved in 21 for $\mathfrak{M}$. It holds for $\mathfrak{M}_{w}$ with the same proof. 
Platek [12] proved that $\mathfrak{M}$ has cardinality $2^{2^{\aleph_{0}}}$ (i.e. the maximum possible for a collection of sets of reals) by showing that $\mathfrak{M}$ has antichains of that cardinality. (He mentions that the result was noted independently by Elisabeth Jockusch and John Stillwell.) In fact, in $\mathfrak{M}$ such large antichains occur in every infinite interval:

Theorem 1.5 (Terwijn [21]). Let $[\mathbf{A}, \mathbf{B}]$ be an interval in $\mathfrak{M}$ with $\mathbf{A}<_{M} \mathbf{B}$. Then either $[\mathbf{A}, \mathbf{B}]$ is isomorphic to the finite Boolean algebra $2^{n}$ for some $n \geqslant 1$, or $[\mathbf{A}, \mathbf{B}]$ contains an antichain of size $2^{2^{\aleph_{0}}}$. In the latter case, it is consistent that it also contains a chain of size $2^{2^{\aleph_{0}}}$.

In particular, $\mathfrak{M}$ 's version of Theorem 1.4 is the only way to generate finite intervals of $\mathfrak{M}$. As we will see in what follows, the situation for $\mathfrak{M}_{w}$ is rather different.

We will use the following theorem at several places below.

Theorem 1.6 (Lachlan and Lebeuf [6]; cf. also Lerman [7, p. 164]). Every countable upper semilattice with a least element is isomorphic to an initial segment of the Turing degrees $\mathcal{D}_{T}$.

\section{More on Chains AND ANTiChains}

Although every countable linear order can be embedded into $\mathfrak{M}_{w}$ (because by Theorem 1.6 this already holds for the Turing degrees), the following result shows that not every countable linear order is isomorphic to an interval in $\mathfrak{M}_{w}$. (From Theorem 3.14 it will follow that every finite linear order is isomorphic to an interval in $\mathfrak{M}_{w}$.)

Proposition 2.1. Not every countable linear order is isomorphic to an interval in $\mathfrak{M}_{w}$.

Proof. Consider the linear order $\omega+\omega^{*}$ (that is, a copy of $\omega$ followed by a reverse copy of $\omega$ ). Suppose that $\mathcal{A}_{n}$ and $\mathcal{B}_{n}, n \in \omega$, are mass problems such that for all $n$ and $m$,

$$
\mathcal{A}_{n}<_{w} \mathcal{A}_{n+1}<_{w} \mathcal{B}_{m+1}<_{w} \mathcal{B}_{m} .
$$

Let $\mathcal{C}=\prod_{m \in \omega} \mathcal{B}_{m} \equiv_{w} \bigcup_{m \in \omega} \mathcal{B}_{m}$. Then for all $n, \mathcal{A}_{n}<_{w} \mathcal{C}<_{w} \mathcal{B}_{n}$, so the interval $\left[\mathcal{A}_{0}, \mathcal{B}_{0}\right]$ is not isomorphic to $\omega+\omega^{*}$.

Proposition 2.2. $\mathfrak{M}_{w}$ contains linear intervals that are countably infinite.

Proof. Consider the linear order $1+\omega^{*}$ (a least element plus a reverse copy of $\omega)$. By Theorem 1.6 we can embed this order in the Turing degrees as an initial segment: Let $\mathcal{F}=\left\{f_{n}: n \in \omega\right\}$ be such that $f_{n+1}<_{T} f_{n}$ and such that

$$
h \leqslant_{T} f_{0} \rightarrow h \text { computable } \vee \exists n h \equiv_{T} f_{n}
$$

for every $h$. Let $\mathcal{B}=\left\{h: h \mathbb{Z}_{T} f_{0}\right\}$ and $\mathcal{A}=\mathcal{B} \times \mathcal{F}$. (Note that $\mathcal{A}$ is in fact w-equivalent to $0^{\prime}$.) Now if $\mathcal{C} \in[\mathcal{A}, \mathcal{B}]$, then $\mathcal{C}$ can be split in disjoint parts $\mathcal{C}_{0}$ and $\mathcal{C}_{1}$ such that $\mathcal{C}_{0}$ is maximal with the property $\mathcal{B} \leqslant_{w} \mathcal{C}_{0}$ and $\left\{f: f \leqslant_{T} f_{0}\right\} \leqslant_{w} \mathcal{C}_{1}$. Then $\mathcal{C}_{1} \subseteq \mathcal{F}$ and $\mathcal{C} \equiv_{w} \mathcal{B} \times \mathcal{C}_{1}$. So it suffices to analyze all subclasses of $\mathcal{F}$ : For every $I \subseteq \omega$ consider $\mathcal{C}_{I}=\mathcal{B} \times\left\{f_{n}: n \in I\right\}$. If $I$ is infinite, then $\mathcal{C}_{I} \leqslant w \mathcal{F}$; hence

\footnotetext{
${ }^{4}$ The precise set-theoretic assumption that is needed is discussed in 21. The existence of such large chains is in fact independent, as follows from results in Baumgartner [1; see also Comfort and Remus [3]. We thank George Barmpalias for pointing out these references to us.
} 
$\mathcal{C}_{I} \equiv_{w} \mathcal{A}$. For $I$ and $J$ finite we have $\mathcal{C}_{I} \leqslant{ }_{w} \mathcal{C}_{J}$ whenever $\min I \leqslant \min J$. So we see that the interval $(\mathcal{A}, \mathcal{B})$ contains only the countably many elements $\mathcal{B} \times\left\{f_{n}\right\}$, $n \in \omega$.

By Proposition 2.2 there are linear nonempty intervals in $\mathfrak{M}_{w}$. This contrasts the situation for $\mathfrak{M}$, where by Theorem 1.5 all the linear intervals are empty. So here we already see that Theorem 1.4 is not the only way anymore to generate finite intervals.

$\mathfrak{M}_{w}$ contains antichains of size $2^{2^{\aleph_{0}}}$, using the same argument that Platek used for $\mathfrak{M}$ (starting with an antichain of size $2^{\aleph_{0}}$ in the Turing degrees, form $2^{2^{\aleph_{0}}}$ incomparable combinations), but Proposition 2.2 shows that they do not occur in every infinite interval, as we had for $\mathfrak{M}$ (cf. Theorem 1.5). In fact there are intervals with maximal antichains of every possible size:

Theorem 2.3. Each of the following possibilities is realized by some interval $[\mathbf{A}, \mathbf{B}]$ in $\mathfrak{M}_{w}$ :

(1) $[\mathbf{A}, \mathbf{B}]$ contains an antichain of size $n$, but not of size $n+1$,

(2) $[\mathbf{A}, \mathbf{B}]$ contains an antichain of size $\aleph_{0}$, but no uncountable antichain,

(3) $[\mathbf{A}, \mathbf{B}]$ contains an antichain of size $2^{\aleph_{0}}$, but not of size $2^{2^{\aleph_{0}}}$,

(4) (Platek [12]) $[\mathbf{A}, \mathbf{B}]$ contains an antichain of size $2^{2^{\aleph_{0}}}$.

Proof (1). This follows from Theorem 1.4.

(2) Let $x_{0}<x_{1}<x_{2}<\ldots$ be an increasing chain of elements in some lattice and let $y_{0}>y_{1}>y_{2}<\ldots$ be a decreasing chain of elements in the same lattice such that $x_{n} \mid y_{n}$ for all $n$. Let $L$ be the free distributive lattice on these sets of elements with an additional least element. Then $L$ is a countable bottomed distributive lattice, so by Theorem 1.6 we have that $L$ is embeddable into the Turing degrees as an initial segment. Let $\left\{f_{n}: n \in \omega\right\}$ and $\left\{g_{n}: n \in \omega\right\}$ be representatives from the image $\operatorname{Im}(L)$ of $L$ corresponding to the sequences $x_{n}$ and $y_{n}$, respectively, so that $\left.f_{i}\right|_{T} g_{j}$ for all $i$ and $j$ and such that for all $n, f_{n}<_{T} f_{n+1}$ and $g_{n+1}<_{T} g_{n}$. Let

$$
\begin{aligned}
\mathcal{B} & =\left\{h: \forall n h \not_{T} f_{n}, g_{n}\right\} \text { and } \\
\mathcal{A} & =\mathcal{B} \times\left\{f_{n}, g_{n}: n \in \omega\right\} .
\end{aligned}
$$

Then every $\mathcal{C} \in[\mathcal{A}, \mathcal{B}]$ can be split as $\mathcal{C} \equiv_{w} \mathcal{C}_{0} \times \mathcal{C}_{1}$, with $\mathcal{C}_{0} \subseteq \mathcal{C}$ maximal with the property that $\mathcal{B} \leqslant{ }_{w} \mathcal{C}_{0}$ and $\mathcal{C}_{1} \subseteq \operatorname{Im}(L)$. Claim: the only elements of $\operatorname{Im}(L)$ that are not in $\mathcal{B}$ are the $f_{n}, g_{n}, n \in \omega$. To see the claim, note that the nonzero elements of $\operatorname{Im}(L)$ are free combinations of the $f_{n}$ and $g_{n}$. Clearly $\mathcal{B}$ is closed under joins. By freeness of $L$ it also easily follows that $\operatorname{Im}(L)-\left\{f_{n}, g_{n}: n \in \omega\right\}$ is closed under meets. Hence $\operatorname{Im}(L) \cap \mathcal{B}$ is closed under meets and joins, and from this it easily follows by induction on the complexity of the elements that every element in $\operatorname{Im}(L)-\left\{f_{n}, g_{n}: n \in \omega\right\}$ is in $\mathcal{B}$. This proves the claim. As a consequence, we have (by maximality of $\mathcal{C}_{0}$ ) that $\mathcal{C}_{1} \subseteq\left\{f_{n}, g_{n}: n \in \omega\right\}$. Now $\operatorname{deg}_{w}(\mathcal{C})$ is determined by $\operatorname{deg}_{w}\left(\mathcal{C}_{1}\right)$ : One easily checks that if $\mathcal{C}, \mathcal{D} \in[\mathcal{A}, \mathcal{B}]$ are split as above as $\mathcal{C} \equiv_{w} \mathcal{C}_{0} \times \mathcal{C}_{1}$ and $\mathcal{D} \equiv_{w} \mathcal{D}_{0} \times \mathcal{D}_{1}$, then $\mathcal{C}_{1} \equiv_{w} \mathcal{D}_{1}$ implies that $\mathcal{C} \equiv_{w} \mathcal{D}$. In its turn, $\operatorname{deg}_{w}\left(\mathcal{C}_{1}\right)$ is determined by the minimal $n$ (if any) such that $f_{n} \in \mathcal{C}_{1}$ and by whether $\mathcal{C}_{1}$ contains infinitely or finitely many $g_{m}$ 's, and in the latter case by the maximal $m$ (if any) such that $g_{m} \in \mathcal{C}_{1}$. So we see that there are only countably many possibilities for the degree of $\mathcal{C}_{1}$, and hence for the degree of $\mathcal{C}$, and hence $[\mathcal{A}, \mathcal{B}]$ is countable.

Now consider the mass problems $\mathcal{C}_{n}=\mathcal{B} \times\left\{f_{n}, g_{n}\right\}$. Clearly $\left.\mathcal{C}_{n}\right|_{w} \mathcal{C}_{m}$ if $n \neq m$. So $[\mathcal{A}, \mathcal{B}]$ is countable and contains an infinite antichain. 
(3) Let $L$ be a countably infinite distributive lattice with a least element and an infinite antichain. By Theorem 1.6, $L$ is embeddable into the Turing degrees as an initial segment. Let $f_{n}, n \in \omega$, be a set of representatives of all the degrees in the image of $L$. Consider the interval $[\mathcal{A}, \mathcal{B}]$, where $\mathcal{B}=\left\{h: \forall n h \mathbb{Z}_{T} f_{n}\right\}$ and $\mathcal{A}=\mathcal{B} \times\left\{f_{n}: n \in \omega\right\}$. Then $[\mathcal{A}, \mathcal{B}]$ contains an infinite antichain of elements of the form $\mathcal{B} \times\{f\}$ because $L$ contains a corresponding infinite antichain. For $I \subseteq \omega$ let $\mathcal{C}_{I}=\mathcal{B} \times\left\{f_{n}: n \in I\right\}$. Then for incomparable sets $I, J \subseteq \omega$ it holds that $\left.\mathcal{C}_{I}\right|_{w} \mathcal{C}_{J}$. So $[\mathcal{A}, \mathcal{B}]$ contains an antichain of size $2^{\aleph_{0}}$. Now if $\mathcal{C} \in[\mathcal{A}, \mathcal{B}]$, then $\mathcal{C} \equiv{ }_{w} \mathcal{C}_{0} \times \mathcal{C}_{1}$, with $\mathcal{C}_{0} \subseteq \mathcal{C}$ maximal with the property that $\mathcal{B} \leqslant{ }_{w} \mathcal{C}_{0}$ and $\mathcal{C}_{1} \subseteq \mathcal{A}$. So the Muchnik degree $\operatorname{deg}_{w}(\mathcal{C})$ of every $\mathcal{C} \in[\mathcal{A}, \mathcal{B}]$ is determined by a countable set $\mathcal{C}_{1}$; hence there are at most $2^{\aleph_{0}}$ many elements in $[\mathcal{A}, \mathcal{B}]$.

(4) We can apply Platek's argument to any interval that contains an antichain of size $2^{\aleph_{0}}$ of singletons: Suppose that the interval $[\mathcal{A}, \mathcal{B}]$ contains the elements $\mathcal{B} \times\left\{f_{\alpha}\right\}, \alpha<2^{\omega}$, such that the $f_{\alpha}$ form an antichain in the Turing degrees. For $I \subseteq 2^{\omega}$ let $\mathcal{C}_{I}=\mathcal{B} \times\left\{f_{\alpha}: \alpha \in I\right\}$. Clearly $\mathcal{C}_{I} \in[\mathcal{A}, \mathcal{B}]$. Now for incomparable sets $I, J \subseteq 2^{\omega}$ it holds that $\left.\mathcal{C}_{I}\right|_{w} \mathcal{C}_{J}$, so it suffices to note that there is an antichain of size $2^{2^{\aleph_{0}}}$ in $\mathcal{P}\left(2^{\omega}\right)$. (For some general notes on chains and antichains we refer to [21].)

From the proof of Theorem 2.3 we can also deduce some consequences for chains in $\mathfrak{M}_{w}$ :

(1) By Theorem 1.4 there are intervals containing chains of size $n$ but not of size $n+1$.

(2) By the proof of item (2), and also Proposition 2.2, there are countable intervals with an infinite chain.

(3) The example of an interval given in the proof of item (3) also contains a chain of size $2^{\aleph_{0}}$, but not of size $2^{2^{\aleph_{0}}}$. This is because $\mathcal{P}(\omega)$ has a chain of size $2^{\aleph_{0}}$, so the same holds with $\omega$ replaced by $\left\{f_{n}: n \in \omega\right\}$. A chain in the interval of item (3) cannot be bigger since the interval itself was of size $2^{\aleph_{0}}$.

(4) It is consistent that $\mathfrak{M}_{w}$ has a chain of size $2^{2^{\aleph_{0}}}$; cf. [21]. The conditions for the existence of chains of size $2^{2^{\aleph_{0}}}$ in $\mathcal{P}\left(2^{\omega}\right)$, in $\mathfrak{M}$, and in $\mathfrak{M}_{w}$ are the same, so as for $\mathfrak{M}$ this is independent; cf. footnote 4 .

\section{The Finite InTERVALs of $\mathfrak{M}_{w}$}

Theorem 3.1 (Sorbi [15, 17]). A countable distributive lattice with 0, 1 is embeddable into $\mathfrak{M}$ (preserving 0 and 1 ) if and only if 0 is meet-irreducible and 1 is join-irreducible.

Sorbi proved Theorem 3.1 by embedding the (unique) countable dense Boolean algebra into $\mathfrak{M}$. Inspection of the proof in [15] shows that this algebra also embeds into $\mathfrak{M}_{w}$, since different elements of the algebra are mapped to mass problems in such a way that each one of them contains functions not T-above any of the functions in the other. In particular every finite distributive lattice is embeddable into $\mathfrak{M}_{w}$. In the following we consider lattices that are isomorphic to an interval of $\mathfrak{M}_{w}$. In Theorem 1.5 we saw that for $\mathfrak{M}$ these were precisely the finite Boolean algebras. Of course no nondistributive lattice can be isomorphic to an interval in $\mathfrak{M}$ or $\mathfrak{M}_{w}$ since both structures are distributive (Medvedev [8]). In this section we characterize the finite intervals of $\mathfrak{M}_{w}$ as a certain subclass of the finite distributive lattices (Theorem 3.14). We start with some illustrative examples. 
Example 3.2. That the diamond lattice is isomorphic to an interval in $\mathfrak{M}_{w}$ was already shown in Theorem 1.4 For later purposes we show that this way of obtaining a diamond is essentially the only way. Suppose that $[\mathcal{A}, \mathcal{D}]$ is an interval in $\mathfrak{M}_{w}$ containing precisely two intermediate elements $\mathcal{B}$ and $\mathcal{C}$, and that $\mathcal{B}$ and $\mathcal{C}$ are incomparable; cf. Figure 2, Then $\mathcal{A}$ and $\mathcal{D}$ do not satisfy property (1.1) of

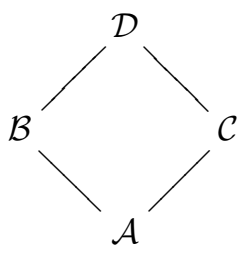

Figure 2

Lemma 1.2 because the interval is finite. So there is a finite set $\mathcal{X} \subseteq \mathcal{A}$ such that $\mathcal{D} \times \mathcal{X} \leqslant{ }_{w} \mathcal{A}$, and hence $\mathcal{D} \times \mathcal{X} \equiv_{w} \mathcal{A}$. Without loss of generality the elements of $\mathcal{X}$ are pairwise T-incomparable and $\{f\} \Varangle_{w} \mathcal{D}$ for every $f \in \mathcal{X}$. Clearly $\mathcal{X}$ must be nonempty since otherwise $\mathcal{A} \equiv_{w} \mathcal{D}$. If $\mathcal{X}$ contains only one element $f$, then for any $\mathcal{Z} \in[\mathcal{A}, \mathcal{D}]$ we have $\mathcal{D} \times\{f\} \leqslant{ }_{w} \mathcal{Z} \leqslant{ }_{w} \mathcal{D}$. If $\{f\} \geqslant_{w} \mathcal{Z}$, then $\mathcal{A} \equiv_{w} \mathcal{Z}$; hence for $\mathcal{Z}>_{w} \mathcal{A}$ we have $\{f\} \neq_{w} \mathcal{Z}$ and hence $\mathcal{D} \times\{f\}^{\prime} \leqslant{ }_{w} \mathcal{Z}$. It follows that $\left[\mathcal{D} \times\{f\}, \mathcal{D} \times\{f\}^{\prime}\right]$ is the initial part of the interval $[\mathcal{A}, \mathcal{D}]$, contradicting that the latter is a diamond. We conclude that $\mathcal{X}$ contains at least two elements. Because the sets $\mathcal{D} \times\{f\}$ with $f \in \mathcal{X}$ are pairwise incomparable elements of $[\mathcal{A}, \mathcal{D}]$ we see that $\mathcal{X}$ can contain at most two elements. So $\mathcal{X}$ contains precisely two elements, $f_{0}$ and $f_{1}$ say. Then $[\mathcal{A}, \mathcal{D}]$ contains the interval $\left[\mathcal{D} \times\left\{f_{0}, f_{1}\right\}, \mathcal{D} \times\left\{f_{0}\right\}^{\prime} \times\left\{f_{1}\right\}^{\prime}\right]$, which by Theorem 1.4 is isomorphic to the diamond lattice. So we must have that $\mathcal{D} \equiv{ }_{w} \mathcal{D} \times\left\{f_{0}\right\}^{\prime} \times\left\{f_{1}\right\}^{\prime}$.

Using similar methods as in the previous example one can show that the following two lattices can be obtained as intervals of $\mathfrak{M}_{w}$ :
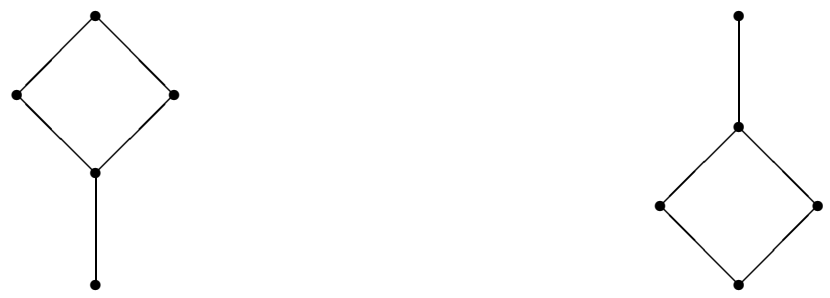

The first one uses an $f$ and two incomparable elements $f_{0}$ and $f_{1}$ that are minimal over $f$; the second one uses incomparable elements $f_{0}$ and $f_{1}$ such that their join $f_{0} \oplus f_{1}$ is minimal over both of them.

Next we show that not every finite distributive lattice is isomorphic to an interval in $\mathfrak{M}_{w}$.

Proposition 3.3. The double diamond lattice from Figure 3 is not isomorphic to an interval in $\mathfrak{M}_{w}$. 


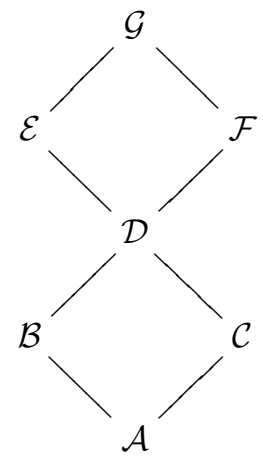

Figure 3

Proof. Assume for a contradiction that the interval $[\mathcal{A}, \mathcal{G}]$ is isomorphic to the lattice of Figure 3, As in Example 3.2 we can argue that there is a finite set $\mathcal{X} \subseteq \mathcal{A}$ such that $\mathcal{A} \equiv_{w} \mathcal{X} \times \mathcal{G}$. Using the same reasoning as before we can argue that $\mathcal{X}$ contains precisely two T-incomparable elements $f_{0}$ and $f_{1}$ with $\left\{f_{0}\right\},\left\{f_{1}\right\} \ngtr_{w} \mathcal{G}$. (If $\mathcal{X}$ contained at least three of such elements, then by Theorem 1.4 the interval $[\mathcal{A}, \mathcal{G}]$ would contain a copy of $2^{3}$, but the interval contains only 7 elements, so this is impossible.) Since by Example 3.2 there is only one way of obtaining a diamond, there are T-incomparable $g_{0}$ and $g_{1}$ with $\left\{g_{0}\right\},\left\{g_{1}\right\} \ngtr_{T} \mathcal{G}$ such that

$$
\begin{aligned}
\mathcal{A} & \equiv_{w} \mathcal{G} \times\left\{f_{0}, f_{1}\right\} \\
\mathcal{B} & \equiv_{w} \mathcal{G} \times\left\{f_{0}\right\}^{\prime} \times\left\{f_{1}\right\} \\
\mathcal{C} & \equiv_{w} \mathcal{G} \times\left\{f_{0}\right\} \times\left\{f_{1}\right\}^{\prime} \\
\mathcal{D} & \equiv_{w} \mathcal{G} \times\left\{f_{0}\right\}^{\prime} \times\left\{f_{1}\right\}^{\prime} \equiv_{w} \mathcal{G} \times\left\{g_{0}, g_{1}\right\} \\
\mathcal{E} & \equiv_{w} \mathcal{G} \times\left\{g_{0}\right\}^{\prime} \times\left\{g_{1}\right\} \\
\mathcal{F} & \equiv_{w} \mathcal{G} \times\left\{g_{0}\right\} \times\left\{g_{1}\right\}^{\prime} \\
\mathcal{G} & \equiv_{w} \mathcal{G} \times\left\{g_{0}\right\}^{\prime} \times\left\{g_{1}\right\}^{\prime} .
\end{aligned}
$$

From the two equations for $\mathcal{D}$ it follows that $\left\{g_{0}, g_{1}\right\}>{ }_{w}\left\{f_{0}, f_{1}\right\}$. Now there are two cases:

- Both $g_{i}$ 's are T-above both $f_{j}$ 's. But then we have

$$
\mathcal{D} \equiv_{w} \mathcal{G} \times\left\{f_{0}\right\}^{\prime} \times\left\{f_{1}\right\}^{\prime} \leqslant{ }_{w} \mathcal{G} \times\left\{f_{0} \oplus f_{1}\right\}<_{w} \mathcal{G} \times\left\{g_{0}, g_{1}\right\} \equiv_{w} \mathcal{D},
$$

a contradiction. (The second to last inequality is strict since $f_{0} \oplus f_{1}<_{T}$ $g_{0}, g_{1}$ because $g_{0}$ and $g_{1}$ are incomparable.)

- The $g_{i}$ 's are not both above $f_{0}$ and $f_{1}$. Hence either there is precisely one $g_{i}$ above each $f_{j}$, or there are precisely two $g_{i}$ 's above one $f_{j}$. In both cases there is at least one $g_{i}$ T-incomparable to an $f_{j}$, say $\left.f_{0}\right|_{T} g_{1}$. Now consider the element $\mathcal{H}=\mathcal{G} \times\left\{f_{0}\right\} \times\left\{g_{1}\right\}^{\prime}$. Clearly $\mathcal{H} \in[\mathcal{A}, \mathcal{G}]$. But $\mathcal{H}$ is w-incomparable to $\mathcal{D}: \mathcal{H} \mathbb{k}_{w} \mathcal{D}$ because $\mathcal{H} \mathbb{K}_{w}\left\{g_{1}\right\}$, and $\mathcal{D} \mathbb{K}_{w} \mathcal{H}$ because $\mathcal{D} \nless_{w}\left\{f_{0}\right\}$. So again we have reached a contradiction, because $[\mathcal{A}, \mathcal{G}]$ does not contain an element incomparable to $\mathcal{D}$.

Since both cases are contradictory we conclude that it is impossible that $[\mathcal{A}, \mathcal{G}]$ is isomorphic to the double diamond. 
We will see later (in Proposition 3.6 and Theorem 3.14) that the double diamond lattice of Figure 3 is the smallest possible counterexample, in the sense that any other counterexample contains it as a sublattice.

Let us recall some elementary lattice theory from Grätzer [5]. Let $L$ be a distributive lattice. $J(L)$ denotes the set of all nonzero join-irreducible elements of $L$. $J(L)$ is a poset under the ordering of $L$. For $a \in L$ define

$$
r(a)=\{x \in J(L): x \leqslant a\} .
$$

For a poset $P$ let $H(P)$ be the collection of downward closed subsets of $P$, partially ordered by inclusion. Then $H(P)$ is a distributive lattice, and we have

Theorem 3.4 ([5, Theorem II.1.9]). For any finite distributive lattice $L$ the mapping $a \mapsto r(a)$ is an isomorphism between $L$ and $H(J(L))$.

Thus the mappings $J$ and $H$ are inverses of each other, and they relate the class of finite distributive lattices with the class of all finite posets.

Say that a lattice $L$ contains another lattice $L^{\prime}$ as a subinterval if there is an interval $[a, b] \subseteq L$ that is isomorphic to $L^{\prime}$. Note that this is not the same as saying that $L^{\prime}$ is a sublattice of $L$. For example, the free distributive lattice on three elements $F_{D}(3)$, depicted in Figure 4, contains the double diamond of Figure 3 as a sublattice, but not as a subinterval.

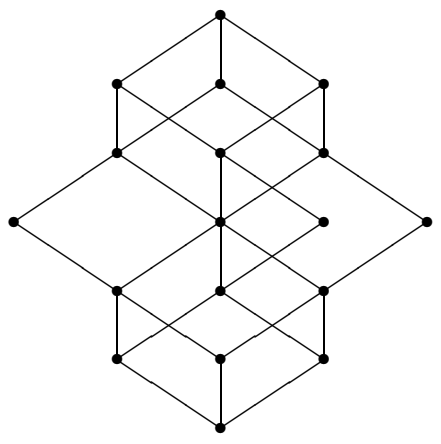

FigurE 4. The free distributive lattice on three elements.

Definition 3.5. We call a finite distributive lattice $L$ double diamond-like if in the poset $J(L)$ there are two incomparable elements with at least two minimal upper bounds.

So if $L$ is double diamond-like, then $J(L)$ contains the configuration from Figure 5. where the lines denote intervals that may contain (possibly incomparable)

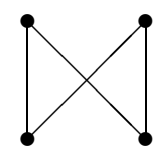

FiguRE 5

other elements. By the next proposition the double diamond lattice is the smallest example of a double diamond-like lattice. 
Proposition 3.6. Any double diamond-like lattice contains the double diamond lattice from Figure 3 as a sublattice.

Proof. If $L$ is double diamond-like, then $J(L)$ contains elements $x_{0}$ and $x_{1}$ that have two minimal upper bounds $y_{0}$ and $y_{1}$. Hence $L$ contains the sublattice generated by $\left\{x_{0}, x_{1}, y_{0}, y_{1}\right\}$, which is the double diamond lattice.

Theorem 3.7. For any finite distributive lattice $L$, the following are equivalent:

(i) $L$ is not double diamond-like,

(ii) the poset $J(L)$ is an initial segment of an upper semilattice,

(iii) $L$ does not have a double diamond-like lattice as a subinterval.

Proof. The equivalence of (i) and (ii) is immediate from Definition 3.5. (iii) trivially implies (i). For the implication from (ii) to (iii), suppose that $J(L)$ is an initial segment of an upper semilattice, and let $[a, b]$ be an interval in $L$. We prove that $[a, b]$ is not double diamond-like. Suppose for a contradiction that $J([a, b])$ contains the configuration of Figure 5, with bottom elements $x_{0}$ and $x_{1}$ and top elements $y_{0}$ and $y_{1}$. We cannot immediately conclude from this that $J(L)$ contains the same configuration, for $J([a, b])$ and $J(L)$ can even be disjoint. Nevertheless, suppose that $y_{0}$ is join-reducible in $L$ as $y_{0}=z_{0}+z_{1}$, with $z_{0} \mid z_{1}$. By Lemma 3.8 we can choose $z_{0}$ and $z_{1}$ such that $z_{0} \times x_{0} \neq z_{0} \times x_{1}$ and $z_{0} \nless y_{1}$. Then in $L$ the set $\left\{z_{0} \times x_{0}, z_{0} \times x_{1}, z_{0}, y_{1}\right\}$, is partially ordered as in Figure 5 . Continuing in this way we can reduce the configuration until the top element $y_{0}$ has become join-irreducible, and of course we can reduce $y_{1}$ in the same way. Then $L$ contains the configuration of Figure 5 with both top elements in $J(L)$. We claim that we can choose maximal join-irreducible elements $v_{0} \leqslant x_{0}$ and $v_{1} \leqslant x_{1}$ such that $v_{0} \nless x_{1}$ and $v_{1} \nless x_{0}$. Namely, if all maximal join-irreducible elements below $x_{0}$ were also below $x_{1}$, then they, and any join of them, would be below $x_{0} \times x_{1}$; hence $x_{0} \leqslant x_{0} \times x_{1} \leqslant x_{1}$, a contradiction. So we can pick $v_{0}$ and $v_{1}$ as above. Since $v_{0}$ and $v_{1}$ are maximal below $x_{0}$ and $x_{1}$, the elements $y_{0}$ and $y_{1}$ are minimal upper bounds of them in $J(L)$. So we see that $L$ contains Figure 5 with all four elements in $J(L)$. But this contradicts that $J(L)$ is an initial segment of an upper semilattice and hence that the bottom two elements $x_{0}$ and $x_{1}$ should have a least upper bound in $J(L)$.

Lemma 3.8. In the proof of Theorem 3.7 above, if $y_{0}=z_{0}+z_{1}$ in $L, z_{0} \mid z_{1}$, then we can choose such $z_{0}$ and $z_{1}$ with $z_{0} \times x_{0} \neq z_{0} \times x_{1}$ and $z_{0} \nless y_{1}$.

Proof. Suppose that $y_{0}=z_{0}+z_{1}$ in $L$, with $z_{0} \mid z_{1}$. Note that $z_{0}$ and $z_{1}$ cannot be both in $[a, b]$ because $y_{0}$ is join-irreducible in $[a, b]$. Suppose that

$$
\forall v, w \in L\left(v \mid w \wedge y_{0}=v+w \rightarrow v, w \notin[a, b]\right) .
$$

Consider $z_{0}$ and $a+z_{1}$. If $a+z_{1}=y_{0}$, then this contradicts (3.1) (because both $a, z_{1}<y_{0}$ they must be incomparable in this case). If $a+z_{1}<y_{0}$, then by $\left(a+z_{1}\right)+z_{0}=y_{0}$ we again contradict (3.1). Hence (3.1) is false, and if $y_{0}=z_{0}+z_{1}$ with $z_{0} \mid z_{1}$ in $L$ we can always choose $z_{0} \notin[a, b]$ and $z_{1} \in[a, b]$. In this case $z_{0}+a=y_{0}$, for if $z_{0}+a<y_{0}$, then by $\left(a+z_{0}\right)+z_{1}=y_{0}$ we would have $y_{0}$ join-reducible in $[a, b]$, a contradiction. Hence for every $c \in\left[a, y_{0}\right]$ it holds that $z_{0}+c=y_{0}$, and in particular,

$$
z_{0}+x_{0}=z_{0}+x_{1}=y_{0} .
$$

Now we also have $z_{0} \nless y_{1}$ because otherwise $y_{0}=z_{0}+x_{0} \leqslant y_{1}$, a contradiction. 
Finally we prove that $z_{0} \times x_{0} \neq z_{0} \times x_{1}$. Suppose that $z_{0} \times x_{0}=z_{0} \times x_{1}$. Because by (3.2) it holds that $z_{0}+x_{0}=z_{0}+x_{1}$, we have

$$
\begin{aligned}
x_{1} & =\left(z_{0} \times x_{1}\right)+x_{1} \\
& =\left(z_{0} \times x_{0}\right)+x_{1} \\
& =\left(z_{0}+x_{1}\right) \times\left(x_{0}+x_{1}\right) \quad \text { (by distributivity) } \\
& =\left(z_{0}+x_{0}\right) \times\left(x_{0}+x_{1}\right) \\
& =x_{0}+\left(z_{0} \times x_{1}\right) \\
& \geqslant x_{0} .
\end{aligned}
$$

From this contradiction we conclude that $z_{0} \times x_{0} \neq z_{0} \times x_{1}$.

Example 3.9. Before giving the general result of how to obtain lattices as intervals of $\mathfrak{M}_{w}$ we give one more specific example to illustrate the method. Figure 6 depicts the procedure to obtain a given lattice $L$ as an interval of $\mathfrak{M}_{w}$. The top left side

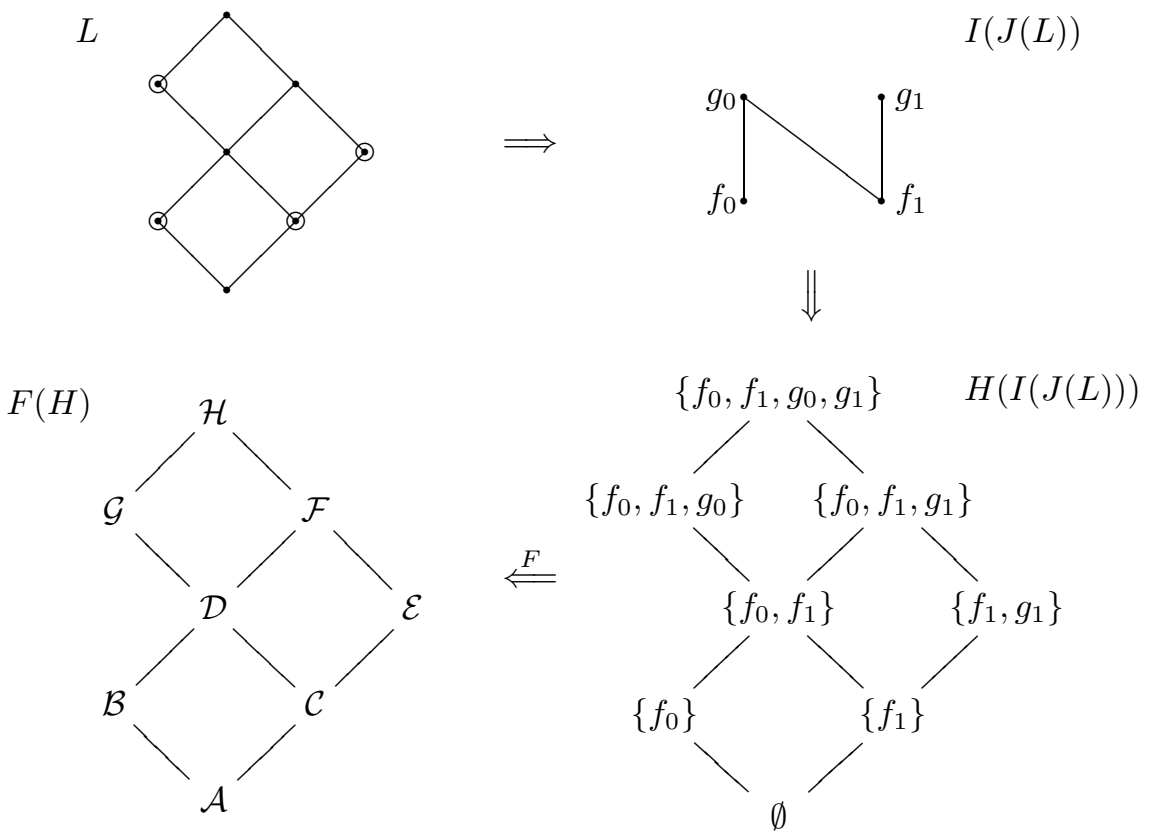

FiguRE 6. Procedure to obtain an interval in $\mathfrak{M}_{w}$ isomorphic to a given $L$.

of the picture shows an example of a finite distributive lattice, with its nonzero join-irreducible elements circled. The partial order $J(L)$ is depicted on the top right. Now for the lattice $L$ in this particular example we can map the poset $J(L)$ to an isomorphic configuration $I(J(L))$ in $\mathcal{D}_{T}$, such that the intervals in $I(J(L))$ contain no other elements than those of $I(J(L))$. (The picture remains the same, so we drew it only once. That one can find this configuration in $\mathcal{D}_{T}$ follows from the general results quoted at the beginning of the proof of Theorem 3.10) This means that the only relations are the ones indicated in the picture, $g_{0}$ covers $f_{0}$ and $f_{1}$, and $g_{1}$ covers $f_{1}$. (Recall that "covers" entails minimality. Also note that for notational simplicity we are identifying $f$ here with $\operatorname{deg}_{T}(f)$.) Next we can form 
the distributive lattice $H=H(I(J(L)))$, which is isomorphic to $L$ by Theorem 3.4 . Finally we apply the mapping $F: H \rightarrow \mathfrak{M}_{w}$ defined as follows. First define

$$
\mathcal{X}=\left\{h>_{T} f_{0}:\left.h\right|_{T} g_{0}\right\} \cup\left\{h>_{T} f_{1}:\left.\left.h\right|_{T} g_{0} \wedge h\right|_{T} g_{1}\right\} .
$$

This has the effect that modulo $\mathcal{X}$ we have $\mathcal{X} \times\left\{f_{0}\right\}^{\prime} \equiv_{w} \mathcal{X} \times\left\{g_{0}\right\}$ (this follows from the minimality of $g_{0}$ over $f_{0}$ ) and $\mathcal{X} \times\left\{f_{1}\right\}^{\prime} \equiv_{w} \mathcal{X} \times\left\{g_{0}, g_{1}\right\}$. For every $A \in H$ define

Finally define

$$
\widehat{A}=\{f \in I(J(L)): f \text { maximal in } A\} .
$$

$$
F(A)=\mathcal{X} \times \prod\left\{\{f\}^{\prime}: f \in \widehat{A}\right\} \times\left\{f \in I(J(L)):\left.f\right|_{T} \widehat{A}\right\} .
$$

Here $\left.f\right|_{T} \widehat{A}$ denotes that $\left.f\right|_{T} g$ for every $g \in \widehat{A}$. We thus obtain the lattice $F(H)$ on the bottom left of the picture, with

$$
\begin{aligned}
\mathcal{A} & =\mathcal{X} \times\left\{f_{0}, f_{1}\right\} \\
\mathcal{B} & =\mathcal{X} \times\left\{f_{0}\right\}^{\prime} \times\left\{f_{1}\right\} \\
\mathcal{C} & =\mathcal{X} \times\left\{f_{0}\right\} \times\left\{f_{1}\right\}^{\prime} \equiv_{w} \mathcal{X} \times\left\{f_{0}, g_{1}\right\} \\
\mathcal{D} & =\mathcal{X} \times\left\{f_{0}\right\}^{\prime} \times\left\{f_{1}\right\}^{\prime} \equiv_{w} \mathcal{X} \times\left\{f_{0}\right\}^{\prime} \times\left\{g_{1}\right\} \equiv_{w} \mathcal{X} \times\left\{g_{0}, g_{1}\right\} \\
\mathcal{E} & =\mathcal{X} \times\left\{f_{0}\right\} \times\left\{g_{1}\right\}^{\prime} \\
\mathcal{F} & =\mathcal{X} \times\left\{f_{0}\right\}^{\prime} \times\left\{g_{1}\right\}^{\prime} \equiv_{w} \mathcal{X} \times\left\{g_{0}\right\} \times\left\{g_{1}\right\}^{\prime} \\
\mathcal{G} & =\mathcal{X} \times\left\{g_{0}\right\}^{\prime} \times\left\{g_{1}\right\} \\
\mathcal{H} & =\mathcal{X} \times\left\{g_{0}\right\}^{\prime} \times\left\{g_{1}\right\}^{\prime} .
\end{aligned}
$$

Using Example 3.2 one can check that $F$ is an isomorphism between $H$ and $F(H)$, so that the interval $[\mathcal{A}, \mathcal{H}]=[F(\emptyset), F(I(J(L)))]$ is indeed isomorphic to $L$.

We are now ready to prove:

Theorem 3.10. Suppose that $L$ is a finite distributive lattice such that $J(L)$ is an initial segment of a finite upper semilattice. Then $L$ is isomorphic to an interval of $\mathfrak{M}_{w}$.

Proof. We follow the procedure depicted in Figure 6. Let $L$ be as in the hypothesis of the theorem. Since $J(L)$ is an initial segment of a finite upper semilattice, by Theorem 1.6 we have a finite poset $I(J(L))$ in $\mathcal{D}_{T}$ that is isomorphic to $J(L)$, with the property that if $g$ covers $f$ in $J(L)$, then the image of $g$ is a minimal cover of $f$ in $\mathcal{D}_{T}$. Furthermore, the minimal elements of $I(J(L))$ can be chosen to be of minimal T-degree (so that in particular they are all noncomputable). Next we form the distributive lattice $H=H(I(J(L)))$, which is isomorphic to $L$ by Theorem 3.4 . Finally we define the mapping $F: H \rightarrow \mathfrak{M}_{w}$ as follows. For a given $f \in I(J(L))$ let $g_{0}, \ldots, g_{m}$ be all elements of $I(J(L))$ covering $f$. Define

$$
\begin{aligned}
\mathcal{X}_{f} & =\left\{h \in \omega^{\omega}: h>\left.\left._{T} f \wedge h\right|_{T} g_{0} \wedge \ldots \wedge h\right|_{T} g_{m}\right\}, \\
\mathcal{X} & =\bigcup_{f \in I(J(L))} \mathcal{X}_{f} .
\end{aligned}
$$

Note that for every $f$ as above we have the properties

$$
\begin{array}{rll}
\mathcal{X} \times\{f\}^{\prime} & \equiv_{w} & \mathcal{X} \times\left\{g_{0}, \ldots, g_{m}\right\}, \\
\mathcal{X} & \Varangle_{w} & \{f\} .
\end{array}
$$


Also notice that if $f$ is maximal in $I(J(L))$ (i.e. $f$ is maximal in the poset as well as $\leqslant_{T}$-maximal), then there are no elements of $I(J(L))$ covering $f$; hence $\mathcal{X}_{f} \equiv_{w}\{f\}^{\prime}$. Furthermore we have for every $h \in \omega^{\omega}$,

$$
\{h\} \geqslant_{w} I(J(L)) \wedge\{h\} \nexists_{w} \mathcal{X} \longrightarrow h \in I(J(L)) .
$$

Next, for every $A \in H$ define

$$
\begin{gathered}
\widehat{A}=\{f \in I(J(L)): f \text { maximal in } A\}, \\
F(A)=\mathcal{X} \times \prod\left\{\{f\}^{\prime}: f \in \widehat{A}\right\} \times\left\{f \in I(J(L)):\left.f\right|_{T} \widehat{A}\right\} .
\end{gathered}
$$

Here $\left.f\right|_{T} \widehat{A}$ denotes that $\left.f\right|_{T} g$ for every $g \in \widehat{A}$. This concludes the definition of the mapping $F$. By definition, $\left.f\right|_{T} \emptyset$ holds for every $f$, so we have that

$$
F(\emptyset)=\mathcal{X} \times I(J(L)) \equiv_{w} \mathcal{X} \times\{f \in I(J(L)): f \text { minimal in } I(J(L))\} .
$$

We thus obtain the lattice $F(H)$. Note that $H$ has $\emptyset$ as least element and $I(J(L))$ as largest element. We prove that $F$ is an isomorphism from $H$ to the interval $[F(\emptyset), F(I(J(L)))] \subseteq \mathfrak{M}_{w}$. Since $H$ is isomorphic to $L$ this suffices to prove the theorem.

$F$ is injective. Suppose that $A, B \in H$. Note that since $A$ and $B$ are downwards closed, $A=B$ if and only if $\widehat{A}=\widehat{B}$. So it suffices to show that if $\widehat{A} \nsubseteq \widehat{B}$, then $F(A) \not \equiv_{w} F(B)$. Suppose that $f \in \widehat{A}-\widehat{B}$. Since $f \in I(J(L))$ we have $\{f\} \nexists_{w} \mathcal{X}$ by (3.4). Since $f \in \widehat{A}$ we also have $\{f\} \Varangle_{w} F(A)$ because $\widehat{A}$ is an antichain. If $\left.f\right|_{T} \widehat{B}$, then $\{f\} \geqslant{ }_{w} F(B)$, so in this case $F(A) \not_{w} F(B)$. If $f \chi_{T} \widehat{B}$, then there is $g \in \widehat{B}$ with either $f \geqslant_{T} g$ or $g>_{T} f$. In the first case we have $f>_{T} g$ (because $g$ is in $\widehat{B}$ and $f$ is not); hence $\{f\} \geqslant{ }_{w} F(B)$, and again we can conclude that $F(A) \not \equiv{ }_{w} F(B)$. In the second case, since $g>_{T} f \in \widehat{A}$ we have $\{g\} \geqslant_{w} F(A)$, but $\{g\} \ngtr_{w} F(B)$ because $g \in \widehat{B}$ and $\widehat{B}$ is an antichain, so again $F(A) \not \equiv_{w} F(B)$.

$F$ is monotone. We claim that $A \subseteq B$ implies that $F(A) \leqslant_{w} F(B)$. Suppose that $A \subseteq B$ and that $h \in F(B)$. We prove that $\{h\} \geqslant_{w} F(A)$. We have the following three cases, corresponding to the three components of $F(B)$ :

- If $h \in \mathcal{X}$, then we are immediately done.

- If $h \in I(J(L)),\left.h\right|_{T} \widehat{B}$, then we have one of the following three options:

$-\left.h\right|_{T} \widehat{A}$. In this case we are done immediately.

- $\exists g \in \widehat{A} h \geqslant_{T} g$. In this case we cannot have $h \in \widehat{A}$ because $A \subseteq B$ and $\left.h\right|_{T} \widehat{B}$, so we have $h>_{T} g$ and hence $\{h\} \geqslant{ }_{w} F(A)$.

$-\exists g \in \widehat{A} \quad h \leqslant_{T} g$. This case cannot occur because $A$ is downwards closed; hence $h$ would be in $A$ and hence in $B$, contradicting $\left.h\right|_{T} \widehat{B}$.

- $h>_{T} f$ for some $f \in \widehat{B}$. When $f \in \widehat{A}$ we are done. If $f \notin \widehat{A}$, then since $A \subseteq B$ and $f$ is maximal in $B$ we have $f \notin A$, so either $\left.f\right|_{T} \widehat{A}$, in which case we are done or $\exists g \in \widehat{A} f \geqslant_{T} g$, in which case $f>_{T} g$ since $f \notin \widehat{A}$, and again we are done.

$F(A \cap B) \equiv_{w} F(A) \times F(B)$ : By monotonicity of $F$ we have $F(A \cap B) \leqslant w$ $F(A), F(B)$, hence also $F(A \cap B) \leqslant{ }_{w} F(A) \times F(B)$. For the other direction $\geqslant_{w}$, suppose that $h \in F(A \cap B)$. We consider the three cases corresponding to the three components of $F(A \cap B)$.

- If $\{h\} \geqslant{ }_{w} \mathcal{X}$, then we immediately have that $\{h\} \geqslant{ }_{w} F(A), F(B)$. 
- Suppose that $h \in F(A \cap B)$ because $h>_{T} f$ for some $f \in \widehat{A \cap B}$. If $h \notin I(J(L))$, then by (3.5) we have $\{h\} \geqslant{ }_{w} \mathcal{X}$, so we may assume that $h \in I(J(L))$. If $\{h\} \geqslant_{w}\left\{g \in I(J(L)):\left.g\right|_{T} \widehat{A}\right\}$ or $\{h\} \geqslant_{w}\{g \in I(J(L)):$ $\left.\left.g\right|_{T} \widehat{B}\right\}$, then we are done. Otherwise, since $h \in I(J(L))$ itself, in particular both $h \chi_{T} \widehat{A}$ and $h \chi_{T} \widehat{B}$, say that $g_{0} \in \widehat{A}$ and $g_{1} \in \widehat{B}$ are such that $h \chi_{T} g_{0}$ and $h \chi_{T} g_{1}$. It is impossible that $h \leqslant_{T} g_{0}, g_{1}$ because then (because $A, B$ are downwards closed) $h \in A \cap B$, contradicting $f \in \widehat{A \cap B}$. So at least one of $g_{0}<_{T} h$ and $g_{1}<_{T} h$ must hold. But in the first case we have $\{h\} \geqslant_{w} F(A)$ and in the second $\{h\} \geqslant_{w} F(B)$.

- Finally suppose that $\left.h\right|_{T} \widehat{A \cap B}$. When $\left.h\right|_{T} \widehat{A}$ or $\left.h\right|_{T} \widehat{B}$, then we are done, so suppose that neither of these hold, say $h \chi_{T} f \in \widehat{A}$ and $h \chi_{T} g \in \widehat{B}$. When either $f$ or $g$ is in $\widehat{A \cap B}$, then $h \chi_{T} \widehat{A \cap B}$ contrary to assumption, so we have that $f, g \notin \widehat{A \cap B}$. When $f \geqslant_{T} g$, then $g \in A \cap B$, and because $g \notin \widehat{A \cap B}$ there exists then $k \in \widehat{A \cap B}$ with $k>_{T} g$, contradicting $g \in \widehat{B}$. Likewise, $g \geqslant_{T} f$ is impossible, so we have $\left.f\right|_{T} g$. Hence either $h>_{T} f, g$ or $h<_{T} f, g$. In the latter case $h \in A \cap B$, contradicting $\left.h\right|_{T} \widehat{A \cap B}$, and in the former case we have $\{h\} \geqslant{ }_{w} F(A), F(B)$.

Hence every $h \in F(A \cap B)$ computes an element of either $F(A)$ or $F(B)$.

$F(A \cup B) \equiv_{w} F(A)+F(B)$ : By monotonicity of $F$ we have $F(A), F(B) \leqslant w$ $F(A \cup B)$, hence also $F(A)+F(B) \leqslant{ }_{w} F(A \cup B)$. For the other direction, suppose that $\{h\} \geqslant_{w} F(A), F(B)$. We prove that $\{h\} \geqslant_{w} F(A \cup B)$. If $\{h\} \geqslant_{w} \mathcal{X}$ we are immediately done, so assume that $\{h\} \Varangle_{w} \mathcal{X}$. We have to prove that either

$$
\begin{gathered}
\exists f \in \widehat{A \cup B} h>_{T} f \\
\text { or } \quad \exists f \in I(J(L))\left[\left.f\right|_{T} \widehat{A \cup B} \wedge h \geqslant_{T} f\right] .
\end{gathered}
$$

We have the following cases, corresponding to the four remaining ways in which $h$ can be above the components of $F(A)$ and $F(B)$ that are different from $\mathcal{X}$ :

- $\{h\} \geqslant_{w}\left\{f \in I(J(L)):\left.f\right|_{T} \widehat{A}\right\},\left\{f \in I(J(L)):\left.f\right|_{T} \widehat{B}\right\}$. Suppose that $h \geqslant_{T} f_{0} \oplus f_{1}, f_{0}, f_{1} \in I(J(L)),\left.f_{0}\right|_{T} \widehat{A}$ and $\left.f_{1}\right|_{T} \widehat{B}$. Note that it is not possible that $f_{0} \oplus f_{1} \leqslant_{T} g$ for some $g \in \widehat{A \cup B}$ because then $f_{0} \oplus f_{1}$ would be below an element of either $\widehat{A}$ or $\widehat{B}$, contradicting that $\left.f_{0}\right|_{T} \widehat{A}$ and $\left.f_{1}\right|_{T} \widehat{B}$. So we either have $f_{0} \oplus f_{1}>_{T} g$ for some $g \in \widehat{A \cup B}$ or $\left.f_{0} \oplus f_{1}\right|_{T} \widehat{A \cup B}$. In the first case we have $h>_{T} g$ and we are done by way of (3.6). In the second case we are done by way of (3.7) because we may assume that $f_{0} \oplus f_{1} \in I(J(L))$. Namely, if $f_{0} \oplus f_{1} \notin I(J(L))$, then by (3.5) we have $\left\{f_{0} \oplus f_{1}\right\} \geqslant_{w} \mathcal{X}$; hence $\{h\} \geqslant_{w} \mathcal{X}$.

- $\{h\} \geqslant_{w}\left\{f \in I(J(L)):\left.f\right|_{T} \widehat{A}\right\}$ and $h>_{T} f_{0}$ for some $f_{0} \in \widehat{B}$. If there is such an $f_{0}$ with $f_{0} \in \widehat{A \cup B}$, then we are done by way of (3.6), so assume without loss of generality that $f_{0} \in \widehat{B}-\widehat{A \cup B}$. As above, by (3.5) we may assume that $h \in I(J(L))$. Hence if $\left.h\right|_{T} \widehat{A \cup B}$, then we are done by (3.7), so assume that $h \chi_{T} \widehat{A \cup B}$, say $g \in \widehat{A \cup B}, h \chi_{T} g$. We have one of the following two cases:

$-h \leqslant_{T} g$. In this case $g \in \widehat{B}$ is impossible by $h>_{T} f_{0} \in \widehat{B}$, so we must have $g \in \widehat{A}$. But this contradicts $\{h\} \geqslant{ }_{w}\left\{f \in I(J(L)):\left.f\right|_{T} \widehat{A}\right\}$.

$-h>_{T} g$. In this case we are done by way of (3.6). 
- $\{h\} \geqslant_{w}\left\{f \in I(J(L)):\left.f\right|_{T} \widehat{B}\right\}$ and $h>_{T} f_{0}$ for some $f_{0} \in \widehat{A}$. This is completely symmetric to the previous case.

- $h>_{T} f_{0}, f_{1}$ for some $f_{0} \in \widehat{A}$ and $f_{1} \in \widehat{B}$. As in previous cases, we may assume by (3.5) that $h \in I(J(L))$. Hence when $\left.h\right|_{T} \widehat{A \cup B}$ we are done by (3.7), so assume that $h \chi_{T} \widehat{A \cup B}$, say $h \chi_{T} f$ with $f \in \widehat{A \cup B}$. Since $f_{0}, f_{1}<_{T} h$ it is impossible that $h \leqslant_{T} f$, for then either $f_{0}$ would not be in $\widehat{A}$ or $f_{1}$ would not be in $\widehat{B}$. Hence $h>_{T} f$, and we are done by way of (3.6).

$F$ is surjective. It remains to check that if $\mathcal{C} \in[F(\emptyset), F(I(J(L)))]$, then there is $A \in H$ such that $F(A) \equiv_{w} \mathcal{C}$. To this end, let $A$ be a maximal subset of $I(J(L))$ such that $\mathcal{C} \geqslant_{w} F(A)$. We claim that then also $\mathcal{C} \leqslant w F(A)$. Namely we have $\mathcal{C} \leqslant{ }_{w} \mathcal{X}$ because $\mathcal{C} \leqslant{ }_{w} F(I(J(L)))$. As for the other components of $F(A)$, suppose that $f \in I(J(L)),\left.f\right|_{T} \widehat{A}$ and suppose that $\mathcal{C}$ contains no element $\leqslant_{T} f$. Let $B$ be the downward closure of $A \cup\{f\}$ in the poset $I(J(L))$. Then $\mathcal{C} \geqslant_{w} F(B)$, for the elements of $\mathcal{C}$ that are mapped to $f$ in the reduction $\mathcal{C} \geqslant_{w} F(A)$ are all $>_{T} f$. But $\mathcal{C} \geqslant{ }_{w} F(B)$ contradicts the maximality of $A$. It follows that $\mathcal{C} \leqslant w\{f \in I(J(L))$ : $\left.\left.f\right|_{T} \widehat{A}\right\}$. We also have $\left.\mathcal{C} \leqslant w \prod\{f\}^{\prime}: f \in \widehat{A}\right\}$. Namely suppose not; that is, suppose there is $f \in \widehat{A}$ such that $\mathcal{C} \mathbb{k}_{w}\{f\}^{\prime}$. Such an $f$ cannot be maximal in $I(J(L))$ because $\mathcal{C} \leqslant_{w} F(I(J(L)))$. Hence the set $\left\{g_{0}, \ldots, g_{m}\right\}$ of all elements of $I(J(L))$ covering $f$ is nonempty. By (3.3) we have $\mathcal{X} \times\{f\}^{\prime} \equiv_{w} \mathcal{X} \times\left\{g_{0}, \ldots, g_{m}\right\}$; hence there is $g \in\left\{g_{0}, \ldots, g_{m}\right\}$ such that $\mathcal{C} \Varangle_{w}\{g\}$. But then it follows from $\mathcal{C} \geqslant_{w} F(A)$ that actually $\mathcal{C} \geqslant_{w} F(A \cup\{g\})$, contradicting the maximality of $A$. Note that the set $A \cup\{g\}$ is downwards closed in $I(J(L))$ since $A$ is downwards closed and $f \in A$. We have thus proved that $\mathcal{C} \equiv_{w} F(A)$. This concludes the proof of the surjectivity of $F$ and of the theorem.

Recall that $C(\mathcal{A})$ denotes the upward closure of $\mathcal{A}$. In Terwijn [19] it was proved that a mass problem $\mathcal{A}$ is join-reducible in $\mathfrak{M}_{w}$ if and only if

$$
\exists g, h \notin C(\mathcal{A})\left[\left.g\right|_{T} h \wedge g \oplus h \in C(\mathcal{A})\right] .
$$

The condition (3.8) already occurred in Sorbi [17] as a sufficient condition for joinreducibility of Medvedev degrees. The condition does not characterize the joinreducible Medvedev degrees [19] 5 The following variant characterizes the joinirreducible elements of an interval in $\mathfrak{M}_{w}$. The proof is almost identical to the proof in 19 .

Proposition 3.11. A mass problem $\mathcal{A}$ is join-reducible in an interval $[\mathcal{X}, \mathcal{Y}] \subseteq \mathfrak{M}_{w}$ if and only if

$$
\exists g, h \notin C(\mathcal{A})\left[\left.g\right|_{T} h \wedge g \oplus h \in C(\mathcal{A}) \wedge g, h \in C(\mathcal{X})\right] .
$$

Corollary 3.12. The meet of two join-irreducible elements in an interval $[\mathcal{X}, \mathcal{Y}] \subseteq$ $\mathfrak{M}_{w}$ is again join-irreducible in $[\mathcal{X}, \mathcal{Y}]$.

Proof. Suppose that $\mathcal{A} \times \mathcal{B}$ is join-reducible in $[\mathcal{X}, \mathcal{Y}]$. By (3.9) there are incomparable $g$ and $h$ such that $g, h \notin C(\mathcal{A} \times \mathcal{B}), g \oplus h \in C(\mathcal{A} \times \mathcal{B})$ and $g, h \in C(\mathcal{X})$. In particular $g, h \notin C(\mathcal{A})$ and $g, h \notin C(\mathcal{B})$, and either $g \oplus h \in C(\mathcal{A})$ or $g \oplus h \in C(\mathcal{B})$. In the first case $\mathcal{A}$ is join-reducible in $[\mathcal{X}, \mathcal{Y}]$, and in the second case $\mathcal{B}$.

Theorem 3.13. If $L$ is isomorphic to a finite interval in $\mathfrak{M}_{w}$, then $J(L)$ is an initial segment of a finite upper semilattice.

\footnotetext{
${ }^{5}$ Note added: Such a characterization was recently given by Shafer 14 .
} 
Proof. Suppose that $L$ is isomorphic to a finite interval $[\mathcal{X}, \mathcal{Y}]$ in $\mathfrak{M}_{w}$, and suppose that $\mathcal{A}$ and $\mathcal{B}$ are incomparable nonzero join-irreducible elements in $[\mathcal{X}, \mathcal{Y}]$. We show that $\mathcal{A}$ and $\mathcal{B}$ do not have two minimal upper bounds in $J(L)$ (hence they have a least upper bound in $J(L)$ in case they have an upper bound at all). Since $L$ is finite, if $\mathcal{A}$ and $\mathcal{B}$ have an upper bound in $J(L)$ they have a minimal upper bound. Suppose $\mathcal{C}$ and $\mathcal{D}$ are two incomparable such upper bounds in $J(L)$. Then by Corollary 3.12 the element $\mathcal{C} \times \mathcal{D}$ is a smaller upper bound in $J(L)$, contradicting the minimality of $\mathcal{C}$ and $\mathcal{D}$. So there can be at most one minimal upper bound in $J(L)$. Hence $J(L)$ is an initial segment of a finite upper semilattice.

By combining the above results we obtain the following characterization of the finite intervals of $\mathfrak{M}_{w}$ :

Theorem 3.14. For any finite distributive lattice $L$ the following are equivalent:

(1) L is isomorphic to an interval in $\mathfrak{M}_{w}$,

(2) $J(L)$ is an initial segment of a finite upper semilattice,

(3) L does not have a double diamond-like lattice as a subinterval.

Proof. Items (2) and (3) are equivalent by Theorem 3.7 Item (2) implies item (1) by Theorem 3.10. Conversely, (1) implies (2) by Theorem 3.13.

Note that since $L$ is finite, the word "finite" in item (2) above can be deleted. It is also equivalent with the statement that $J(L)$ completed with a greatest element is an upper semilattice.

Corollary 3.15. A finite distributive lattice is an initial segment of $\mathfrak{M}_{w}$ if and only if it has no double diamond-like subinterval and it has a meet-irreducible 0.

Proof. We can extend the definition of the mapping $F$ in the proof of Theorem 3.14 as follows. Define $\mathcal{X}_{f}$ as before and let

$$
\begin{aligned}
& \mathcal{X}_{0}=\left\{h \in \omega^{\omega}:\left.h\right|_{T} f \text { for all } f \text { minimal in } I(J(L))\right\}, \\
& \mathcal{X}=\mathcal{X}_{0} \cup \bigcup_{f \in I(J(L))} \mathcal{X}_{f} .
\end{aligned}
$$

Then for every $A \in H$ define $F(A)$ as before, using this new definition of $\mathcal{X}$. This addition does not change anything in the proof of Theorem 3.10, but now we have that $F(\emptyset) \equiv{ }_{w} 0^{\prime}$, as is easily checked, using that we chose the minimal elements of $I(J(L))$ of minimal T-degree. Thus we obtain that a finite distributive lattice has no double diamond-like subinterval if and only if it is isomorphic to an interval of the form $\left[\mathbf{0}^{\prime}, \mathbf{A}\right]$ in $\mathfrak{M}_{w}$. From this the corollary follows immediately.

It may be noted that the results of this paper in fact hold in greater generality than we have stated them in. Start with a partial order $(P, \leqslant)$ and consider the lattice (in fact, Heyting algebra) of all upwards closed subsets of $P$, ordered by $\supseteq$. The main results of this paper hold for any lattice obtained in this way, provided that every countable upper semilattice with a least element is embeddable in $P$ as an initial segment. (For the characterization from Theorem 3.14 it even suffices that every finite upper semilattice is embeddable in $P$ as an interval.) However, our first interest here is in lattices from computability theory such as the Medvedev and Muchnik lattices, also because of the intriguing connections with constructive logic. For more on this we refer the reader to [18] or [20]. 


\section{ACKNOWLEDGMENTS}

The author thanks Andrea Sorbi for helpful discussions, and George Barmpalias for comments on 21].

\section{REFERENCES}

[1] James E. Baumgartner, Almost-disjoint sets, the dense set problem and the partition calculus, Annals of Mathematical Logic 10 (1976) 401-439. MR0401472 (53:5299)

[2] Stephen Binns and Stephen G. Simpson, Embeddings into the Medvedev and Muchnik lattices of $\Pi_{1}^{0}$ classes, Archive for Mathematical Logic 43 (2004) 399-414. MR2052891(2004m:03156)

[3] W. Wistar Comfort and Dieter Remus, Long chains of Hausdorff topological group topologies, Journal of Pure and Applied Algebra 70 (1991) 53-72. MR:1100505 (92f:22001)

[4] Elena Z. Dyment, Certain properties of the Medvedev lattice, Mathematics of the USSR Sbornik 30 (1976) 321-340. English translation. MR0432433 (55:5421)

[5] George Grätzer, General lattice theory, Second edition, Birkhäuser, 2003. MR.1670580 (2000b:06001)

[6] Alistair H. Lachlan and R. Lebeuf, Countable initial segments of the degrees of unsolvability, Journal of Symbolic Logic 41 (2) (1976), 289-300. MR0403937 (53:7746)

[7] Manuel Lerman, Degrees of unsolvability, Springer, 1983. MR708718 (85h:03044)

[8] Yuri T. Medvedev, Degrees of difficulty of the mass problems, Dokl. Akad. Nauk. SSSR 104(4) (1955) 501-504. MR0073542 (17:448b)

[9] Albert A. Muchnik, Negative answer to the problem of reducibility in the theory of algorithms, Dokl. Akad. Nauk. SSSR (N.S.) 108 (1956) 194-197.

[10] Albert A. Muchnik, On strong and weak reducibility of algorithmic problems, Sibirsk. Math. Zh. 4 (1963) 1328-1341. (In Russian) MR0200151(34:50)

[11] Piergiorgio Odifreddi, Classical recursion theory, Vol. 1, Studies in logic and the foundations of mathematics, Vol. 125, North-Holland, 1989. MR982269 (90d:03072)

[12] Richard A. Platek, A note on the cardinality of the Medvedev lattice, Proc. Amer. Math. Soc. 25 (1970), 917. MR0262080(41:6690)

[13] Hartley Rogers, Jr., Theory of recursive functions and effective computability, McGraw-Hill, 1967. MR0224462 (37:61)

[14] Paul Shafer, A characterization of the join-irreducible Medvedev degrees, (abstract), in: Computability in Europe, CiE 2009, Lecture Notes in Computer Science 5635, p. 353.

[15] Andrea Sorbi, Some remarks on the algebraic structure of the Medvedev lattice, Journal of Symbolic Logic 55(2) (1990) 831-853. MR1056392 (91c:03038)

[16] Andrea Sorbi, Embedding Brouwer algebras in the Medvedev lattice, Notre Dame Journal of Formal Logic 32(2) (1991) 266-275. MR.1123000 (92j:03056)

[17] Andrea Sorbi, The Medvedev lattice of degrees of difficulty, In: S. B. Cooper, T. A. Slaman, and S. S. Wainer (eds.), Computability, Enumerability, Unsolvability: Directions in Recursion Theory, London Mathematical Society Lecture Notes 224, Cambridge University Press, 1996, 289-312. MR1395886 (97g:03048)

[18] Andrea Sorbi and Sebastiaan A. Terwijn, Intermediate logics and factors of the Medvedev lattice, Annals of Pure and Applied Logic 155 (2008) 69-85. MR2455558 (2009j:03066)

[19] Sebastiaan A. Terwijn, Constructive logic and the Medvedev lattice, Notre Dame Journal of Formal Logic 47(1) (2006) 73-82. MR2211183 (2007h:03077)

[20] Sebastiaan A. Terwijn, Constructive logic and computational lattices, habilitation thesis, Technical University of Vienna, 2007.

[21] Sebastiaan A. Terwijn, On the structure of the Medvedev lattice, Journal of Symbolic Logic 73(2) (2008) 543-558. MR2414464 (2009c:03041)

Department of Mathematics, Radboud University Nijmegen, P.O. Box 9010, 6500 GL NiJmegen, the Netherlands

E-mail address: terwijn@math.ru.nl 